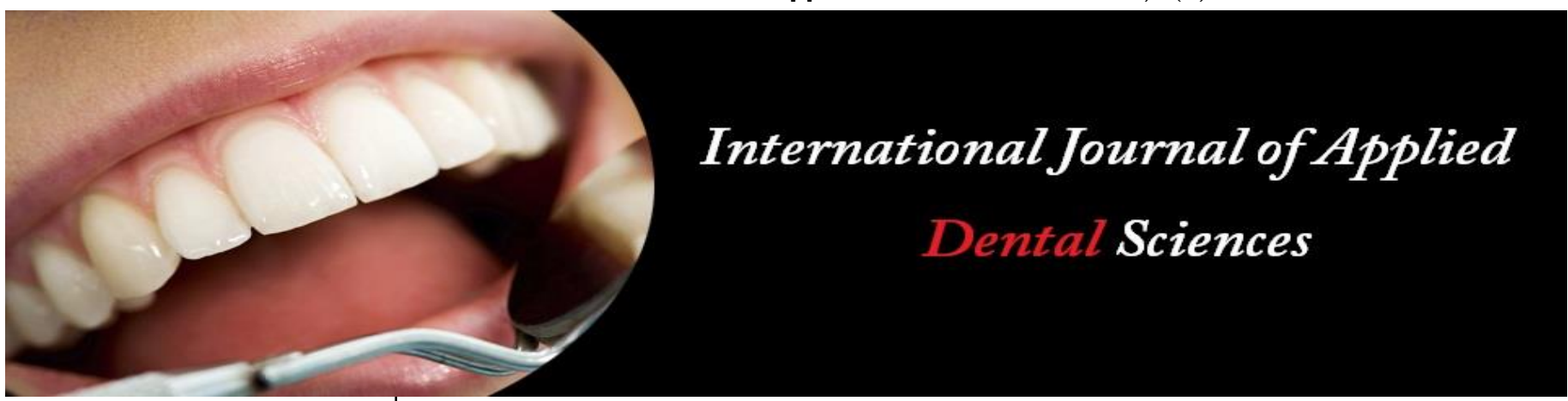

ISSN Print: 2394-7489

ISSN Online: 2394-7497

IJADS 2021; 7(3): 462-469

(C) 2021 IJADS

www.oraljournal.com

Received: 16-05-2021

Accepted: 18-06-2021

Seyedeh Fatemeh Seyed-Javadi

Limoodi

D.D.S, Tehran University of

Medical Sciences, School of

Dentistry, Tehran, Iran

Farshad Khosraviani

D.D.S, University of California

Los Angeles, School of Dentistry,

California, USA

Negar Moghaddasi

D.D.S, Shiraz University of

Medical Sciences, School of

Dentistry, Shiraz, Iran

Shiva Pouya

D.D.S, Tabriz University of

Medical Sciences, School of

Dentistry, Tabriz, Iran

Corresponding Author:

Seyedeh Fatemeh Seyed-Javadi

Limoodi

D.D.S, Tehran University of

Medical Sciences, School of

Dentistry, Tehran, Iran

\section{Effectiveness of photodynamic therapy with curcumin against Candida species: A systematic review}

\section{Seyedeh Fatemeh Seyed-Javadi Limoodi, Farshad Khosraviani, Negar Moghaddasi and Shiva Pouya}

DOI: $\underline{\text { https://doi.org/10.22271/oral.2021.v7.i3g.1337 }}$

\section{Abstract}

Introduction: In recent years, photodynamic therapy (PDT) has shown an effective role in controlling fungal infections such as oral candidiasis both in laboratory and clinical settings. Curcumin as a natural photosensitizer has antibacterial as well as antifungal effects. The aim of the present study was to evaluate the anti-Candida spp. effects of PDT by using curcumin.

Methods: First, the intended keywords were searched in the four following databases: Google Scholar, PubMed, Scopus and Web of Science. The titles were then merged, and the original eligible articles published by the end of 2020 were scrutinized.

Results: Out of 167 merged titles, 12 articles (In-vitro, in-vivo: mice \& G. mellonella) met the required criteria and were included in the study. The studied Candida spp. were $C$. albicans, $C$. dubliniensis, $C$. glabrata and C. tropicalis. Concentrations of curcumin used in the studies ranged from 0.005 to 1000 $\mu \mathrm{M}$. The light sources included LED $(440-460 \mathrm{~nm})$, diode laser $(405,532,650 \mathrm{~nm})$ and Xenon lamp $(370-680 \mathrm{~nm})$. The results of all studies showed that PDT reduced the colony count and the level of Candida spp. metabolism significantly. PDT efficacy was dependent on curcumin concentration, Candida spp., energy and wavelength of radiations, and it proved more effective in planktonic environments than biofilm environments.

Conclusion: PDT using curcumin in a concentration-dependent manner has a potent lethal effect against some Candida spp. and may be considered as a major or adjuvant treatment for some Candida infections.

Keywords: curcumin, photodynamic therapy, laser, Candida, fungus

\section{Introduction}

Fungal diseases are common treatment problems ${ }^{[1]}$. It is estimated that over one billion skin fungal infections and up to 200,000 cases of oral fungal infections may be observed around the world each year ${ }^{[1]}$. C. albicans and to a lesser extent $C$. glabrata, $C$. tropicalis, $C$. parapsilosis and $C$. krusei cause more than $20 \%$ of fungal infections ${ }^{[1-4]}$. Diabetes, using denture, antibiotic consumption, radiation therapy, chemotherapy and immunodeficiency disorders are some of the risk factors for Candida spp. infection ${ }^{[1,5-7]}$. Increased drug resistance among fungal species, the increasing prevalence of infection with non-C. albicans species, an increase in chronic diseases such as diabetes and immunodeficiency disorders and difficulty in managing invasive candidiasis are important challenges in treating patients with fungal infections [1,8]. These challenges necessitate new therapeutic approaches and the production of agents that counteract with Candida resistance ${ }^{[4,9]}$. Up to $9.5 \%$ of Candida species are resistant to fluconazole, and up to $3 \%$ are resistant to next-line drugs such as voriconazole. Unfortunately, resistance to these drugs is higher among non- $C$. albicans species ${ }^{[4]}$, which adds to the existing concerns.

Photodynamic therapy (PDT) is an evolving treatment. PDT was used in the early 20th century to treat skin tumors. Over the past two decades, renewed attention to its cytotoxic effects has opened new, inexpensive, safe, non-invasive or minimally invasive therapeutic methods [10] Treatment of diseases such as periodontitis ${ }^{[11]}$, healing of chronic wounds ${ }^{[12]}$, accelerating the healing of oral mucositis in cancer patients undergoing chemoradiotherapy are of its therapeutic applications ${ }^{[12]}$. In addition, PDT can show a good efficacy close to that of antiCandida drugs, which is a significant success ${ }^{[13]}$. 
PDT is based on the production of free radicals by photosensitizers (PSs). So far, various PSs have been introduced and efforts are underway to produce and strengthen their structures so that they can penetrate the cell and have a good ability to absorb light and produce free radicals [14-17]. The most common PSs used against microorganisms include methylene blue, toluidine blue and rose Bengal dyes ${ }^{[15]}$.

Curcumin (Cur) has recently been introduced as a natural PS against microorganisms. Cur is the most important active polyphenol in the rhizome of Curcuma longa plant. The yellow powder of the rhizome of this plant is used as a flavor and coloring food, especially in Asian countries ${ }^{[18]}$. Cur could potentially play an effective role in controlling the pathology of some diseases and in maintaining health. Modulation of memory impairment and oxidative stress [19], anti-cancer effects, modulation of disorders related to metabolic syndrome and modulation of inflammation are among its therapeutic effects ${ }^{[20]}$. Cur reacts with proxy species at low oxygen pressures, and is capable of producing and absorbing free radicals. In addition, induction of cellular apoptosis, dimer formation and maintaining its structure in reaction with proxy species increase Cur's advantage as a PS ${ }^{[22,23]}$. In laboratory reports Cur has shown antifungal effects ${ }^{[24]}$, and its use as PDT has increased such effects.

This study aimed to investigate the role of PDT by using Cur in the control of different Candida species in a systematic review study. A recent review study evaluated the effect of curcumin activation by light-emitting diode (LED) irradiation on Streptococcus mutans and Candida albicans, and did not evaluate the other Candida species and light sources such as laser ${ }^{[23]}$, which could change the efficacy of PDT. The present study can provide a better conclusion of the available evidence in this regard.

\section{Methods}

This study was designed to evaluate the published articles on the anti-Candida effects of PDT by using Cur. The reporting framework was based on the Preferred Reporting Items for Systematic Reviews and Meta-Analyses (PRISMA) checklist [24]. This checklist is designed for clinical trial studies but it can be used for other studies as well.

First, the following keywords were gathered based on MeSH and keywords in related articles.

(Laser OR "Low Power Laser" OR "Low Level Laser" OR "High Power Laser" OR "Photodynamic Therapy") AND (Candida OR "Candida albicans" OR Candidiasis OR Antifungal OR Fungus) AND (Curcumin OR Turmeric OR "Curcuma Longa")

The keywords were then searched in three databases: PubMed, Scopus, and Web of Science (WOS). Search results were limited to original English-language articles published by the end of 2020 using options available on the website of each database. Also, to extend the search scope, the keywords were searched in Google Scholar and the first 200 titles displayed were examined, and qualified studies were included in the study ${ }^{[25]}$.

The titles were then merged into Mendeley desktop software (ver. 1.19) to remove duplicates. The remaining titles were submitted electronically to two reviewers (S.S, N.M). The reviewers reviewed the submitted articles (abstract-text) and classified them according to the study criteria.

\subsection{Inclusion criteria are as follows}

- Original English language articles

- Use of Cur as PS

- PDT intervention

- Using Candida spp. in studies

\subsection{Exclusion criteria are as follows}

- Lack of control groups

- Use of Cur derivatives or its modified compositions

- Flaws in the methodology or the results

- Lack of access to the full text of articles

If the two reviewers did not agree on some of the selected studies, a third reviewer (S.P) decided on them. Final articles were submitted to a fourth reviewer (F.K) to be reviewed and reported with precision.

From the articles, information including type of study, type of light source, optical wavelength, energy and power, Cur concentration, presence of other PSs, type of Candida species and fungicidal evaluation methods were extracted.

The results of Cur effectiveness in combination with light irradiation were reported as effective if there was a significant difference compared to the positive control group (or no significant difference with the negative control group) and ineffective if there was no significant difference with the positive control group.

To the best of our knowledge, there is no checklist to assess the quality and risk of bias in laboratory studies. Accordingly, the use of inclusion and exclusion criteria and the focus on studies' methodologies can prevent the selection of laboratory studies with high risk of bias.

\section{Results}

\subsection{Search results}

A search through the above four databases yielded 167 titles. After integrating them into Mendeley software, 118 titles remained. The titles then, were sent to the two reviewers for screening the articles based on the study criteria. 96 articles were excluded from the study, the reasons for which are shown in Figure 1. 22 titles had inclusion criteria, from which 10 articles were excluded for reasons such as adding a nanoparticle to Cur and changing its structure. Also, a clinical trial study was excluded from the study due to the lack of a control group. Finally, 12 studies were reviewed from which, information was extracted carefully. Table 1 shows a summary of the final articles. 


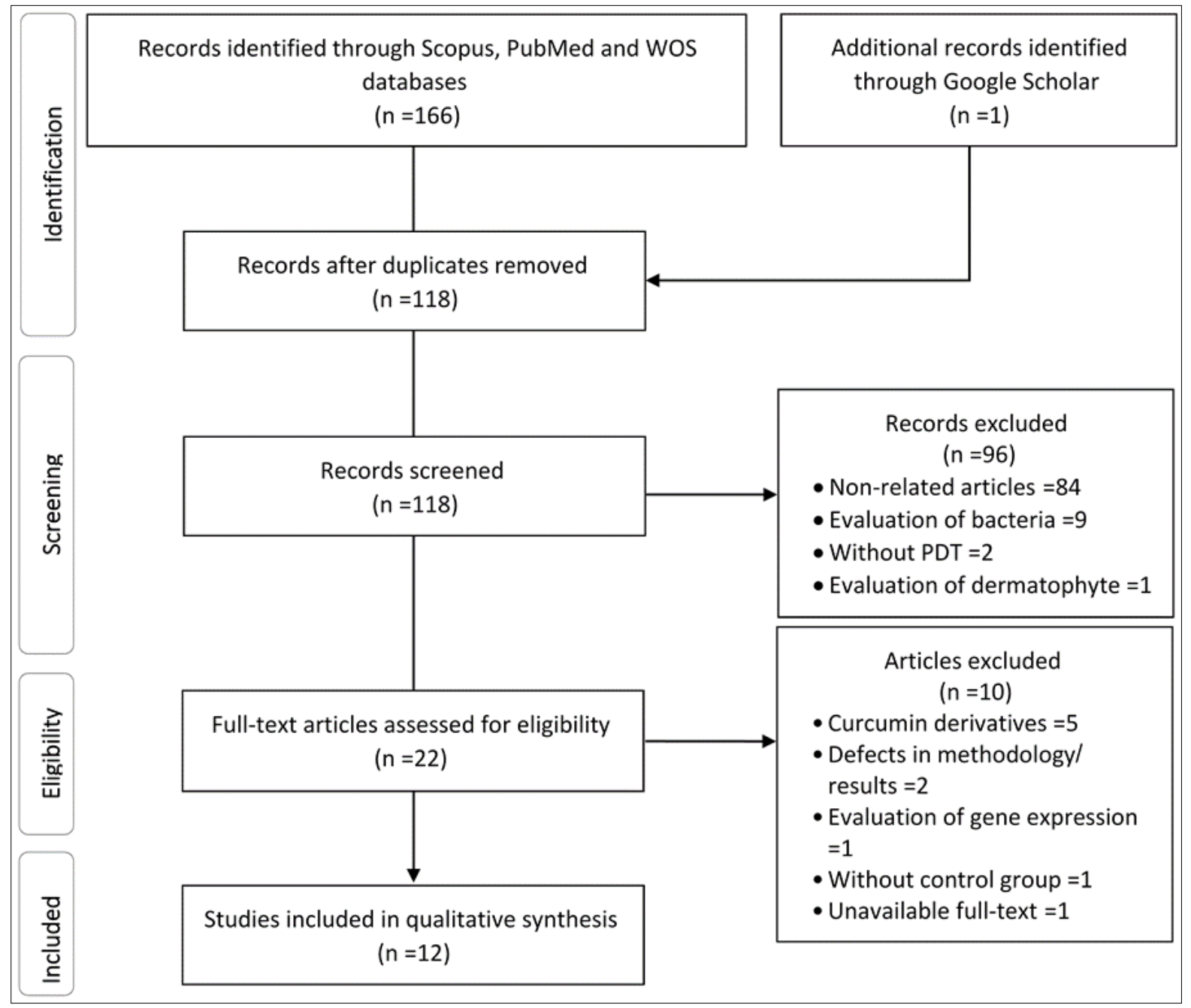

Fig 1: Articles search result

Table 1: A summary of the final studies

\begin{tabular}{|c|c|c|c|c|c|c|c|c|c|c|}
\hline Author & Study type & Microorganisms & Sample & \begin{tabular}{|c|}
$\begin{array}{c}\text { Light } \\
\text { source }\end{array}$ \\
\end{tabular} & \begin{tabular}{|c|}
$\begin{array}{c}\text { Wavelength } \\
(\mathbf{n m})\end{array}$ \\
\end{tabular} & $\begin{array}{l}\text { energy } \\
(\mathrm{J} / \mathrm{cm} 2)\end{array}$ & \begin{tabular}{|c|} 
power \\
$(\mathbf{m W} / \mathbf{c m} 2)$
\end{tabular} & $(\mu \mathrm{M}) \mathbf{P S}$ & evaluation & efficacy \\
\hline \begin{tabular}{|c|} 
Dovigo et \\
al., $2011^{[26]}$
\end{tabular} & In-vitro & C. albicans & ATCC 90028 & LED & $440-460$ & $1.32-37.5$ & 22 & Cur,0.005-20 & $\begin{array}{c}\mathrm{CFU} / \mathrm{ml} \\
\mathrm{XTT}\end{array}$ & $\mathrm{E}$ \\
\hline $\begin{array}{c}\text { Dovigo et } \\
\text { al., } 2011 \text { [27] }\end{array}$ & In-vitro & $\begin{array}{l}\text { C. albicans } \\
\text { C. tropicalis } \\
\text { C. glabrata }\end{array}$ & clinical & LED & $440-460$ & $\begin{array}{c}5.28,18, \\
25.5,37.5\end{array}$ & 22 & Cur,5, 10, 20 & $\begin{array}{c}\mathrm{CFU} / \mathrm{ml} \\
\mathrm{XTT} \\
\mathrm{CV}\end{array}$ & $\mathrm{E}$ \\
\hline \begin{tabular}{|c|} 
Dovigo et \\
al., 2013 [28]
\end{tabular} & $\begin{array}{l}\text { In-vivo, } \\
\text { mice }\end{array}$ & C. albicans & ATCC 90028 & LED & $440-460$ & 37.5 & 89.2 & Cur,20, 40, 80 & $\mathrm{CFU} / \mathrm{ml}$ & $\mathrm{E}$ \\
\hline $\mid \begin{array}{c}\text { Andrade } e t \\
\text { al., } 2013 \text { [29] }\end{array}$ & In-vitro & $\begin{array}{c}\text { C. albicans } \\
\text { C. glabrata } \\
\text { C. dubliniensis }\end{array}$ & \begin{tabular}{|c|} 
ATCC 90028 \\
ATCC 2001 \\
CBS 7987 \\
\end{tabular} & LED & $440-460$ & 5.28 & 22 & $\begin{array}{c}\text { Cur,5, 10, } \\
20,30,40\end{array}$ & $\begin{array}{c}\mathrm{CFU} / \mathrm{ml} \\
\mathrm{XTT}\end{array}$ & $\mathrm{E}$ \\
\hline$\left|\begin{array}{c}\text { Quishida et } \\
\text { al., } 2016^{[30]}\end{array}\right|$ & In-vitro & $\begin{array}{l}\text { C. albicans } \\
\text { C. glabrata } \\
\text { S. mutans }\end{array}$ & \begin{tabular}{|c|} 
ATCC 90028 \\
ATCC 2001 \\
ATCC \\
25175 \\
\end{tabular} & LED & $440-460$ & 37.5 & 22 & $\begin{array}{c}\text { Cur, } 80,100, \\
120\end{array}$ & $\begin{array}{c}\mathrm{CFU} / \mathrm{ml}, \\
\mathrm{XTT} \\
\text { CV }\end{array}$ & $\mathrm{E}$ \\
\hline $\mid \begin{array}{c}\text { Merigo et } \\
\text { al., } 2017[31]\end{array}$ & $\begin{array}{c}\text { In-vitro } \\
\text { In-vivo, } G . \\
\text { mellonella }\end{array}$ & C. albicans & SC5314 & $\begin{array}{l}\text { Diode } \\
\text { laser }\end{array}$ & $\begin{array}{c}405,532 \\
650\end{array}$ & $10,20,30$ & - & \begin{tabular}{|c|} 
Cur, 100 \\
Erythrosine, 100 \\
Toluidine blue, \\
10
\end{tabular} & $\begin{array}{c}\text { An area of } \\
\text { growth, } \\
\text { Survival } \\
\text { rate }\end{array}$ & $\mathrm{E}$ \\
\hline $\begin{array}{c}\text { Al-Asmari } \\
\text { et al., } 2017 \\
{[32]}\end{array}$ & In-vitro & \begin{tabular}{|c|} 
Aspergillus niger \\
Aspergillus flavus \\
Penicillium \\
griseofulvum, \\
Penicillium \\
chrysogenum, \\
Fusarium oxysporum \\
C. albicans \\
Zygosaccharomyces \\
bailii \\
\end{tabular} & $\begin{array}{c}\text { ATCC } 6275 \\
\text { ATCC } 9643 \\
\text { ATCC } 48927 \\
\text { ATCC } 1010 \\
\text { ATCC } 62606 \\
\text { ATCC } 10231 \\
\text { N ATCC } \\
42476\end{array}$ & $\begin{array}{c}\text { Xenon } \\
\text { lamp }\end{array}$ & $370-680$ & $0.24-360$ & $500 \mathrm{w}$ & Cur,100-1000 & $\mathrm{CFU} / \mathrm{ml}$ & $\mathrm{E}$ \\
\hline Sanita $e t$ & In-vitro & C. dubliniensis & CBS 7987 & LED & $440-460$ & 5.28 & 22 & Cur,5,10,20, & $\mathrm{CFU} / \mathrm{mL}$ & $\mathrm{E}$ \\
\hline
\end{tabular}




\begin{tabular}{|c|c|c|c|c|c|c|c|c|c|c|}
\hline al., $2018^{[33]}$ & & & Clinical & & & & & 30,40 & XTT & \\
\hline \begin{tabular}{|c|} 
Hsieh et al., \\
$2018^{[34]}$
\end{tabular} & In-vitro & C. albicans & ATCC 90029 & LED & $440-460$ & 9 & 22 & $\begin{array}{c}\text { Cur, } 1,5,10 \\
20,40,80 \\
\end{array}$ & $\mathrm{CFU} / \mathrm{mL}$ & $\mathrm{E}$ \\
\hline \begin{tabular}{|c|} 
da Silva et \\
al., 2019 [35]
\end{tabular} & In-vitro & C. albicans & ATCC 18804 & LED & $440-460$ & 67 & 21.1 & Cur, $1.5 \%$ & $\mathrm{CFU} / \mathrm{mL}$ & $\mathrm{E}$ \\
\hline $\begin{array}{c}\text { Ma et al., } \\
2019 \\
{[36]}\end{array}$ & In-vitro & C. albicans & $\begin{array}{c}\text { ATCC } 90028 \\
\text { Clinical }\end{array}$ & LED & $440-460$ & $\begin{array}{c}2.6,5.4, \\
7.9,10.7 \\
13.2\end{array}$ & 22 & $\begin{array}{c}\text { Cur,20, 40, 60, } \\
80, \\
100 \\
\end{array}$ & XTT & $\mathrm{E}$ \\
\hline $\mid \begin{array}{c}\text { Rocha } \text { et } \\
\text { al., } 2020^{[37]}\end{array}$ & In-vitro & $\begin{array}{c}\text { E. coli } \\
\text { MRSA } \\
\text { C. albicans } \\
\end{array}$ & $\begin{array}{l}\text { ATCC } 25922 \\
\text { ATCC 33591 } \\
\text { ATCC 90029 } \\
\end{array}$ & LED & $440-460$ & $10.8,32.4$ & 18 & Cur,30,60 & $\mathrm{CFU} / \mathrm{mL}$ & $\mathrm{E}$ \\
\hline
\end{tabular}

\subsection{Type of studies}

All the 12 reviewed studies were performed In-vitro or on animal, and no human clinical trials were observed. In addition to the culture medium, two studies evaluated the intervention on the tongue and oral mucosa of mice ${ }^{[28]}$ and $G$. Mellonella larvae [31]. In the studies, the preparation and culture of Candida spp. were done in suspension (planktonic) and solid media (biofilm). Five studies used only biofilm to evaluate the findings ${ }^{[28,30,35-37]}$.

\subsection{Type of microorganisms and their isolation}

In 11 studies, standard pre-existing laboratory species were used. In three studies, samples of Candida spp. were provided from the human oral mucosa including people with AIDS, lichen planus and oral candidiasis $[27,33,36]$. Six studies used only $C$. albicans to evaluate the antifungal effects of PDT ${ }^{[26,}$ 28, 31, 35-37]. In addition to $C$. albicans, two studies used bacteria including Streptococcus mutans, E. coli, and Methicillin-resistant Staphylococcus aureus (MRSA) [30, 37] One study used seven fungal samples including Candida albicans, Aspergillus niger, Aspergillus flavus, Penicillium griseofulvum, Penicillium chrysogenum, Fusarium oxysporum and Zygosaccharomyces bailii ${ }^{[32]}$. C. dubliniensis was used in two studies ${ }^{[29,33]}$. C. glabrata and C. tropicalis were other studied species of Candida ${ }^{[27,29,30]}$.

\subsection{Types of PS}

Cur was used as PS in 12 studies. Methylene blue and erythrosine were also used in one study ${ }^{[31]} .10$ studies used low concentrations of Cur $(0.005-100 \mu \mathrm{M})$ and one study used concentrations of 100-1000 $\mu \mathrm{M}$ Cur ${ }^{[32]}$. In one study, Cur concentration was reported as $1.5 \mathrm{~g} / \mathrm{Lit}{ }^{[35]}$. Toluidine blue with a concentration of $10 \mu \mathrm{M}$ and erythrosine with a concentration of $100 \mu \mathrm{M}$ were used in one study ${ }^{[31]}$.

\subsection{Light source and radiation conditions}

The LED was irradiated in ten studies with a wavelength range of 420-460nm. One study used the diode lasers with three wavelengths of 405, 532 and $650 \mathrm{~nm}{ }^{[31]}$. One study used a Xenon lamp with a radiation spectrum of $370-680 \mathrm{~nm}$ [32].

In the LED group, the radiation power densities were 18, 21.1, 22 and $89 \mathrm{mw} \mathrm{/} \mathrm{cm}^{2}$, and the energy density of radiations varied from 1.32 to $37.5 \mathrm{~J} / \mathrm{cm}^{2}$. In the laser groups, the power density of radiation was not reported and the radiation energy densities were 10,20 and $30 \mathrm{~J} / \mathrm{cm}^{2}{ }^{[31]}$. In the Xenon lamp group, the radiant power was $500 \mathrm{~W}$, and its radiation energy densities were 24.5 and $73.2 \mathrm{~J} / \mathrm{cm}^{2}{ }^{[32]}$.

\subsection{Investigation of anti-Candida effects}

Colony count (CFU / mL) was used in nine studies; assessment of the level of cellular metabolism by the XTT assay was done in six studies, and growth inhibition zone in the biofilm was used in one study ${ }^{[31]}$. Also, two studies used crystal violet staining and optical spectrometry to investigate the lethal effect as well as the reduction of microorganisms' attachment to the wall of the culture dishes ${ }^{[27,30]}$. Five studies simultaneously used colony count and examined the level of cell metabolism by the XTT assay [26, 27, 29, 30, 33].

\subsection{Effectiveness}

In all studies, PDT, compared to the positive control group, showed a significant result on reducing colony counts, metabolism and attachment of Candida spp., other fungal species and bacteria in both biofilm and planktonic environments. In nine studies, in some settings, the reduction of $C$. albicans, $C$. dubliniensis and $C$. tropicalis reached $100 \%$ which occurred mainly in the planktonic environment [26, 27, 29-32, 34]. C. glabrata showed less sensitivity to PDT and their removal was incomplete.

All three light sources interacted well with Cur and their radiation wavelengths were shown effective. In a histological study, partial elimination of Candida albicans and modulation of inflammation were seen in mice tongue ${ }^{[28]}$. In addition, PDT increased the survival of the infected larvae with $C$. albicans ${ }^{[31]}$.

In the studies, in general, a significant and direct relationship between fungicidal effect and Cur concentration, radiation energy and contact time with Cur before irradiation was recorded. The following is a summary of the reviewed studies. In their three studies, Dovigo et al. investigated the optimal doses of Cur on different laboratory and clinical species of Candida. In addition, investigations were made on the effect of PDT with the help of Cur on the treatment of oral candidiasis of mice ${ }^{[26-28]}$.

First, they investigated the effect of $0-20 \mu \mathrm{M}$ Cur with a maximum energy of $37.5 \mathrm{~J} / \mathrm{cm}^{2}$ and a contact time with Cur of 0-20 minutes on the $C$. albicans. Concentrations of 5, 10, $20 \mu \mathrm{M}$ had the most lethal effect. At a concentration of 20 $\mu \mathrm{M}$ and energy of $5.28 \mathrm{~J} / \mathrm{cm}^{2}$ (4 minutes irradiation) complete lethality was achieved, and the increase of irradiation time had no significant effect. In the biofilm environment, based on the results of planktonic environment, concentrations of 5, $10,20,30,40 \mu \mathrm{M}$, energy of $5.28 \mathrm{~J} / \mathrm{cm}^{2}$ and contact time of 0-20 minutes were used. The contact time of 1 to 20 minutes before irradiation was able to reduce the colony count. The increase of contact time showed a better effect in lethality. The best results were obtained with 20 minutes of contact time, $20 \mu \mathrm{M}$ Cur and a radiation intensity of $5.28 \mathrm{~J} / \mathrm{cm}^{2}[26]$. In another study of theirs, $20 \mu \mathrm{M}$ Cur among concentrations of $5,10,20$, and $80 \mu \mathrm{M}$ and a radiant energy of 5.28 and $18 \mathrm{~J}$ $/ \mathrm{cm}^{2}$ among radiation energies of 5.28, 18, 25.5 and $37.5 \mathrm{~J} /$ $\mathrm{cm}^{2}$ showed the best results on the reduction of $C$. albicans, C. tropicalis and C. glabrata colonies in the planktonic environment. In the planktonic environment, a concentration of $20 \mu \mathrm{M}$ and a minimum energy of $18 \mathrm{~J} / \mathrm{cm}^{2}$ completely killed $C$. tropicalis. Not all of the above concentrations and energies had a complete lethal effect on C. glabrata in the 
planktonic medium. Cur by concentrations of 20, 30 and 40 $\mu \mathrm{M}$ were evaluated in a biofilm medium. After biofilm formation, the above concentrations were contacted with the culture medium for 20 minutes, and then an LED with an energy of 5.28 and $18 \mathrm{~J} / \mathrm{cm}^{2}$ was radiated. The reduction of metabolism in biofilm for $C$. albicans, $C$. tropicalis and $C$. glabrata was $85.3 \%, 80.1 \%$ and $42 \%$ respectively. Also, the reduction rates of cell adhesion to the plastic dish wall for the above Candida spp. were 53.2\%, $69.1 \%$ and $64.1 \%$ respectively ${ }^{[27]}$.

Dovigo et al. reported the effectiveness of PDT with Cur in the mice model as well. Oral mucosa of the mice was infected with $C$. albicans under immunosuppressive conditions, and Cur was exposed to oral mucosa and tongue at concentrations of 20,40 , and $80 \mu \mathrm{M}$, and after 20 minutes, an LED light was radiated. Radiation continued for 7 minutes at a total dose of $37.5 \mathrm{~J} / \mathrm{cm}^{2}$. All PDT settings significantly reduced $C$. albicans' colony. There were dose-dependent effects among which $80 \mu \mathrm{M}$ showed the best effect ${ }^{[28]}$.

Andrade et al. evaluated Cur pre-irradiation contact times (1, 5, 10, and $20 \mathrm{~min}$ ) in planktonic and biofilm media for $C$. albicans, $C$. glabrata, and dubliniensis. One minute contact at $20 \mu \mathrm{M}$ Cur completely reduced $C$. dubliniensis, however, it reduced $C$. albicans and $C$. glabrata by about $85 \%$. Also, in the planktonic environment, all species were completely inactivated by PDT with time contacts of 5, 10 and 20 min and at a concentration of $20 \mu \mathrm{M}$ and no significant difference was observed between the contact times before the above irradiation. For biofilm, concentrations were of 10, 20, 30 and $40 \mu \mathrm{M}$, and irradiation times were 4 and 8 minutes, and contact times before irradiation were $1,5,10$ and 20 minutes. In the biofilm, both the concentration and pre-irradiation times acted independently on lethality. A concentration of 40 $\mu \mathrm{M}$ and a time of 20 minutes had the most lethal effect on the species. Radiation or Cur alone did not prove effective ${ }^{[29]}$.

Quishida et al. analyzed the effect of PDT on the biofilms of C. albicans, C. glabrata and Streptococcus mutans 24 or 48 hours after biofilm formation. They used concentrations of 80 , 100 and $120 \mu \mathrm{M}$ of Cur. In the first 24 hours, C. albicans showed a significant response to PDT with all Cur concentrations without significant differences. In the measurement after 48 hours, only PDT with $120 \mu \mathrm{M}$ Cur had a significant efficacy on $C$. albicans. In the first 24 hours, Cur of 100 and $120 \mu \mathrm{M}$ were deadly for the $C$. albicans alone, but these concentrations were not ineffective after 48 hours. All concentrations of PDT at all times showed a significant inhibitory effect for C. glabrata and S. mutans ${ }^{[30]}$.

Merigo et al. had a different study. They examined different wavelengths of diode laser and three types of PS of $100 \mu \mathrm{M}$ Erythrosine, $10 \mu \mathrm{M}$ toluidine blue, and $100 \mu \mathrm{M}$ curcumin on $C$. albicans in $G$. mellonella larvae and In-vitro settings. The wavelengths used were 405, 532 and $650 \mathrm{~nm}$ and the radiation energies were 10,20 and $30 \mathrm{~J} / \mathrm{cm}^{2}$. Without PS there was no lethal effect in both solid and suspension media. Cur, toluidine blue and erythrosine had the best effect in the suspension, respectively. Only PDT with Cur showed 100\% lethal effect, which was seen in all radiation energies. The highest degree of inhibition was observed at $405 \mathrm{~nm}$ and the use of Cur at any concentration. None of the PSs had a lethal effect on larvae and were safe. PDT significantly increased larvae survival with all PSs ${ }^{[31]}$.

In the study of Alasmari et al. Cur contact with the environment was done 10-30 minutes before or just before the Xenon lamp irradiation on a variety of fungi. Cur concentration of $100-1000 \mu \mathrm{M}$ and radiant energies of 0.24 ,
48, 72 and $96 \mathrm{~J} / \mathrm{cm}^{2}$ were used in the suspension medium. The best response was obtained at a concentration of 600$1000 \mu \mathrm{M}$ Cur, energy of 72 and $96 \mathrm{~J} / \mathrm{cm}^{2}$ and an irradiation time of 6 to 8 minutes. All doses and energies were effective on C. albicans and had $100 \%$ lethality. In biofilm, Cur at 800 $\mu \mathrm{M}$ and 96,240 and $360 \mathrm{~J} / \mathrm{cm}^{2}$ were able to completely inhibit $C$. albicans. The time of exposure to curcumin before irradiation did not affect the lethality significantly ${ }^{[32]}$.

Sanitá et al. evaluated PDT on C. dubliniensis. Samples were obtained from HIV-infected individuals as well as standard laboratory samples. Cur concentrations included 20,30, and $40 \mu \mathrm{M}$, the contact time was $20 \mathrm{~min}$, and radiation energy was $5.28 \mathrm{~J} / \mathrm{cm}^{2}$. Concentration of $20 \mu \mathrm{M}$ in the planktonic environment completely inhibited $C$. dubliniensis. In the biofilm environment, PDT had a significant inhibitory effect at all three concentrations. 30 and $40 \mu \mathrm{M}$ Cur alone without radiation also reduced the viability. In the planktonic medium, contact at 5 and 20 minutes produced similar light absorption, but in biofilm, at 5 minutes, only some parts of the culture medium began to absorb light ${ }^{[33]}$.

Hsieh et al. investigated the effect of PDT or fluconazole or their combination on $C$. albicans. Cur concentrations in this study were $1,5,10,20,40$ and $80 \mu \mathrm{M}$; contact time was 20 minutes, and radiation energy was $9 \mathrm{~J} / \mathrm{cm}^{2}$. In the planktonic environment, concentrations of $1 \mu \mathrm{M}$ and $9 \mathrm{~J} / \mathrm{cm}^{2}$ had a relative lethal effect, and a concentration of $5 \mu \mathrm{M}$ had complete lethality. In the planktonic environment fluconazole caused a significant decrease in $C$. albicans but in culture medium this decrease was not significant. PDT effectively reduced $C$. albicans in biofilm, and when combined with fluconazole, better significant results were obtained. Within 48 hours, the combination of PDT and fluconazole reduced the colony count to $5 \%$, while fluconazole alone reduced the colony count to $20 \%{ }^{[34]}$.

Da Silva et al. examined PDT on bovine rib specimens infected with $C$. albicans. PDT showed a significant lethal effect compared to Cur or light alone and the control group. Cur or light alone was not significantly different from the positive control group ${ }^{[35]}$.

Ma et al. examined PDT on $C$. albicans extracted from oral mucosa of AIDS and lichen planus patients as well as standard laboratory samples. The effectiveness of PDT on the standard sample groups was higher than that of lichen planus and AIDS patients $(90.9 \%, 86.7 \%$ and $66.4 \%)$. Also, a concentration-dependent effect was observed, and the concentration of $60 \mu \mathrm{M}$ was optimal ${ }^{[36]}$.

Rocha et al. evaluated the efficacy of mouthwash containing 30 and $60 \mu \mathrm{M}$ Cur with PDT on C. albicans, MRCA and E. coli biofilms. Settings included 10 minutes of irradiation $10.8 \mathrm{~J} / \mathrm{cm}^{2}$ and 30 minutes of irradiation-32.4 J/ $\mathrm{cm}^{2}$. In the findings, $60 \mu \mathrm{M}$ Cur -30 minutes of irradiation caused $89.4 \%$ reduction in $C$. albicans colony. Cur with a concentration of $30 \mu \mathrm{M}$ decreased the MRCA depending on the irradiation time. For E. coli, the result was similar to MRCA, and the colony growth was reduced by almost $100 \%$, but no timedependent effect was observed ${ }^{[37]}$.

\section{Discussion}

The findings of twelve reviewed articles showed that Cur in combination with light radiation with three sources of LED, diode laser and Xenon lamp played an effective role in reducing Candida spp. in both in-vivo and In-vitro models, and no placebo effect was observed.

The mechanism(s) of PDT and its cellular effects are not understood completely. The production of reactive oxygen 
species (ROS) as a cellular-damaging agent may be the mechanism justifying the effect of PDT ${ }^{[27]}$. The decrease in the metabolism of Candida spp. after PDT in the reviewed studies can be caused by a series of damage to the cell wall, the cell membrane and the genetic content due to ROS [26,36, ${ }^{38,39]}$ as well as cell membrane damage due to Cur toxicity ${ }^{[40]}$. The cell wall of the fungus reduces its permeability and increases cell strength and cell attachment. Cell wall is the first barrier to Cur penetration into the fungus cell ${ }^{[41]}$, and the interaction of Cur with the cell wall can be the first site of PDT damage.

In the reviewed articles PDT was used with 0.005-1000 $\mu \mathrm{M}$ curcumin and its antifungal results was dependent on Cur concentration $[26-28,30,32-34,36,37]$. It seems that in the suspension medium the minimum effective concentration of Cur is 5 to $20 \mu \mathrm{M}$ and in the biofilm, the $20 \mu \mathrm{M}$ and more appropriately the $30-40 \mu \mathrm{M}$ concentrations have a better ability to reduce Candida spp. ${ }^{[26-28,33,34]}$. In addition to Cur concentration, three factors including Candida species, wavelength and radiant energy also affect the results of PDT. In four studies, PDT had a $100 \%$ reducing effect on $C$. albicans, C. dubliniensis, and C. tropicalis ${ }^{[27,29,31,32]}$, but this effect was not complete on $C$. glabrata ${ }^{[27,29]}$. Although $C$. glabrata has more resistance than C. albicans in the clinic ${ }^{[42]}$, but the present finding could be a bias because in other studies, for $C$. glabrata the minimum inhibitory concentration of Cur was higher than C. albicans and C. tropicalis ${ }^{[43,44]}$, and with increasing the concentration of Cur during PDT, its relative resistance may decrease ${ }^{[43,44]}$. Samples collected in the clinic may be more resistant to PDT than the pre-existing standard Candida spp. ${ }^{[36]}$. Various standard species had also been used in studies that may affect their sensitivity to PDT.

In some of the reviewed studies, the increase in irradiation time or energy was mostly associated with the fungicidal effects $[26,27,32,36,37]$. In the biofilm, the radiation energy of 18-37.5 J / $\mathrm{cm}^{2}$ seems to be effective, although a lower radiation energy is required in the suspension medium. Also, the effect of curcumin concentration can be more important than irradiation time or energy ${ }^{[26,29]}$.

In the studies, the biofilm had a weaker response to PDT than the suspension medium. Due to the greater thickness of the biofilm, there are two items that can maintain the effect of PDT relative to the suspension medium. The first is an increase in the concentration of Cur. And the second is the contact time of Cur with the biofilm before irradiation. In the latter case, the contact time provides an opportunity for Cur to penetrate into the deeper layers. According to the results, the Cur contact time, at least 5 minutes for the suspension medium and at least 20 minutes for the biofilm medium, as seen in the clinic, is the ideal time for the anti-Candida effects of PDT ${ }^{\text {[29] }}$

Cur has an absorption spectrum of 270 to $590 \mathrm{~nm}$, and ideally, an absorption spectrum of 410 to $450{ }^{[32,39]}$. This spectrum is clearly existent in the wavelength of LED lamp (440-460nm), Xenon lamp (370-680nm) and also the wavelength of $405 \mathrm{~nm}$ diode laser compared to the other two wavelengths (532, $650 \mathrm{~nm}$ ) in the study of Merigo et al. ${ }^{[31]}$. Therefore, the use of light sources corresponding to the maximum absorption wavelength of Cur can bring about more successful results. Unlike LED and Xenon lamps, lasers produce photons of the same phase, energy, and direction. In other PDT studies with LED or laser, similar results have been shown to reduce bacterial ${ }^{[45,46]}$ and fungal colonies ${ }^{[47]}$. It seems that the type of PS and radiation setting are more important than the type of light sources according to the PS absorption spectrum.
Lasers with and without PDT have shown different antifungal effects. In the study of Najafi et al. $940 \mathrm{~nm}$ diode laser radiation alone had an increasing or neutral effect on $C$. albicans colony ${ }^{[48]}$. In the study of Wiench et al., the $635 \mathrm{~nm}$ diode laser alone is ineffective but PDT with toluidine blue significantly reduces all three species of $C$. albicans, $C$. glabrata, and $C$. krusei ${ }^{[49]}$. In the present reviewed study, three radiation wavelengths of 650, 405 and $532 \mathrm{~nm}$ of diode laser and LED alone, generally had no effect, but in combination with Cur (superior result), erythrosine and toluidine blue, it showed a fungicidal effect ${ }^{[28,30-32]}$ which is similar to findings of the other studies ${ }^{[43,50-52]}$.

In a reviewed study, the effect of fluconazole on biofilm was less than that of PDT, and the combination of the two increased the antifungal effect ${ }^{[34]}$. As in other studies, Cur had a weaker effect on Candida spp. than azoles, nystatin and amphotericin $\mathrm{B}^{[50,52-54]}$. Cur in combination with fluconazole and amphotericin $\mathrm{B}^{[44,50]}$ reduces the MIC of fluconazole and amphotericin B and enhances their effectiveness ${ }^{[34,44]}$. Cur also can sensitize fluconazole-resistant $C$. albicans species ${ }^{[53]}$ which adds to its advantage as a PS.

In summary, the findings of the reviewed articles showed that Cur as PS could play a fungicidal role after light irradiation on Candida spp., and the results generally depend on the concentration of Cur, the type of Candida spp. and the radiation wavelength. Further studies are needed to achieve the ideal radiation settings, especially Cur-mediated laser radiation, as well as clinical effects. To the best of our knowledge, Cur is a safe substance in the usual dosage ${ }^{[55,56]}$, and its topical administration also increases its safety due to minimizing systemic effects.

\section{Funding}

This research received no specific grant from any funding agency in the public, commercial or not-for-profit sectors.

\section{Conflict of interest}

The authors declare that they have no conflict of interest.

\section{Reference}

1. Bongomin F, Gago S, Oladele RO, Denning DW. Global and multi-national prevalence of fungal diseases-estimate precision. Journal of Fungi 2017, 3.

2. Mahmoudi Rad M, Zafarghandi S, Abbasabadi B, Tavallaee M. The epidemiology of Candida species associated with vulvovaginal candidiasis in an Iranian patient population. Eur J Obstet Gynecol Reprod Biol 2011;155(2):199-203.

3. Guinea J. Global trends in the distribution of Candida species causing candidemia, Clinical Microbiology and Infection 2014;20:5-10.

4. Pfaller MA, Diekema DJ, Gibbs DL, Newell VA, Ellis D, Tullio $\mathrm{V}$ et al. Results from the artemis disk global antifungal surveillance study, 1997-2007: A 10. 5-year analysis of susceptibilities of Candida species to fluconazole and voriconazole as determined by CLSI standardized disk diffusion. J Clin Microbiol 2010;48(4):1366-77.

5. Raber-Durlacher JE, Elad S, Barasch A. Oral mucositis, Oral Oncology 2010;46:452-6.

6. Mushi MF, Bader O, Taverne-Ghadwal L, Bii C, Groß U, Mshana SE. Oral candidiasis among African human immunodeficiency virus-infected individuals: 10 years of systematic review and meta-analysis from sub-Saharan Africa, Journal of Oral Microbiology 2017, 9. 
7. Prakash B, Shekar M, Maiti B, Karunasagar I, Padiyath S. Prevalence of Candida spp. Among healthy denture and nondenture wearers with respect to hygiene and age. J Indian Prosthodont Soc 2015;15(1):29-32.

8. Friedman DZP, Schwartz IS. Emerging fungal infections: New patients, new patterns, and new pathogens Journal of Fungi 2019, 5.

9. Bondaryk M, Kurzạtkowski W, Staniszewska M. Antifungal agents commonly used in the superficial and mucosal candidiasis treatment: Mode of action and resistance development, Postepy Dermatologii i Alergologii 2013;30:293-301.

10. Chilakamarthi U, Giribabu L. Photodynamic Therapy: Past, Present and Future. Chemical Record 2017.

11. Sgolastra F, Petrucci A, Gatto R, Marzo G, Monaco A. Photodynamic therapy in the treatment of chronic periodontitis: A systematic review and meta-analysis, Lasers in Medical Science 2013;28:669-82.

12. Pires Marques EC, Piccolo Lopes F, Nascimento IC, Morelli J, Pereira MV, Machado Meiken VM et al. Photobiomodulation and photodynamic therapy for the treatment of oral mucositis in patients with cancer. Photodiagnosis Photodyn Ther 2020, 29.

13. Javed F, Samaranayake LP, Romanos GE. Treatment of oral fungal infections using antimicrobial photodynamic therapy: A systematic review of currently available evidence, Photochemical and Photobiological Sciences 2014;13:726-34.

14. Wijesiri N, Yu Z, Tang H, Zhang P. Antifungal photodynamic inactivation against dermatophyte Trichophyton rubrum using nanoparticle-based hybrid photosensitizers. Photodiagnosis Photodyn Ther 2018;23:202-8.

15. Ghorbani J, Rahban D, Aghamiri S, Teymouri A, Bahador A. Photosensitizers in antibacterial photodynamic therapy: an overview. Laser Ther 2018;27(4):293-302.

16. Zhang Y, Zheng K, Chen Z, Chen J, Hu P, Cai L et al. Rapid killing of bacteria by a new type of photosensitizer. Appl Microbiol Biotechnol 2017;101(11):4691-700.

17. Zhang J, Jiang C, Figueiró Longo JP, Azevedo RB, Zhang H, Muehlmann LA. An updated overview on the development of new photosensitizers for anticancer photodynamic therapy, Acta Pharmaceutica Sinica B 2018;8:137-46.

18. Priyadarsini KI. The chemistry of curcumin: From extraction to therapeutic agent, Molecules 2014;19:20091-112.

19. Effect of curcumin on memory impairment: A systematic review.

20. Hewlings S, Kalman D. Curcumin: A Review of Its Effects on Human Health. Foods 2017;6(10):92.

21. Fujisawa $\mathrm{S}$, Atsumi $\mathrm{T}$, Ishihara $\mathrm{M}$, Kadoma $\mathrm{Y}$. Cytotoxicity, ROS-generation Activity and Radicalscavenging Activity of Curcumin and Related Compounds. Anticancer Res 2004;24(2 B):563-9.

22. Walters DK, Muff R, Langsam B, Born W, Fuchs B. Cytotoxic effects of curcumin on osteosarcoma cell lines. Invest New Drugs 2008;26(4):289-97.

23. Picco D de CR, Cavalcante LLR, Trevisan RLB, SouzaGabriel AE, Borsatto MC, Corona SAM. Effect of curcumin-mediated photodynamic therapy on Streptococcus mutans and Candida albicans: A systematic review of in vitro studies, Photodiagnosis and
Photodynamic Therapy 2019;27:455-61.

24. Liberati A, Altman DG, Tetzlaff J, Mulrow C, Gøtzsche PC, Ioannidis JPA et al. The PRISMA statement for reporting systematic reviews and meta-analyses of studies that evaluate health care interventions: explanation and elaboration. In: Journal of clinical epidemiology 2009.

25. Khosraviani F, Saberi-Demneh A, Asadollahifar R, Nakhostin A, Khazaei P. Post-operative pain management with meloxicam: a systematic literature review in the field of dentistry. Oral Surg 2020;13(2):188-96.

26. Dovigo LN, Pavarina AC, Ribeiro APD, Brunetti IL, Costa CADS, Jacomassi DP et al. Investigation of the photodynamic effects of curcumin against Candida albicans. Photochem Photobiol 2011;87(4):895-903.

27. Dovigo LN, Pavarina AC, Carmello JC, MacHado AL, Brunetti IL, Bagnato VS. Susceptibility of clinical isolates of Candida to photodynamic effects of curcumin. Lasers Surg Med 2011;43(9):927-34.

28. Dovigo LN, Carmello JC, De Souza Costa CA, Vergani CE, Brunetti IL, Bagnato VS et al. Curcumin-mediated photodynamic inactivation of Candida albicans in a murine model of oral candidiasis. Med Mycol 2013;51(3):243-51.

29. Andrade MC, Ribeiro APD, Dovigo LN, Brunetti IL, Giampaolo ET, Bagnato VS et al. Effect of different preirradiation times on curcumin-mediated photodynamic therapy against planktonic cultures and biofilms of Candida spp. Arch Oral Biol 2013.

30. Quishida CCC, De Oliveira Mima EG, Jorge JH, Vergani CE, Bagnato VS, Pavarina AC. Photodynamic inactivation of a multispecies biofilm using curcumin and LED light. Lasers Med Sci 2016.

31. Merigo E, Conti S, Ciociola T, Fornaini C, Polonelli L, Lagori $\mathrm{G}$ et al. Effect of different wavelengths and dyes on Candida albicans: In vivo study using Galleria mellonella as an experimental model. Photodiagnosis Photodyn Ther 2017;18:34-8.

32. Al-Asmari F, Mereddy R, Sultanbawa Y. A novel photosensitization treatment for the inactivation of fungal spores and cells mediated by curcumin. J Photochem Photobiol B Biol 2017.

33. Sanita PV, Pavarina ACC, Dovigo LNLN, Ribeiro APD, Andrade MC, de Oliveira Mima EG et al. Curcuminmediated anti-microbial photodynamic therapy against Candida dubliniensis biofilms. Lasers Med Sci 2018;33(4):709-17.

34. Hsieh Y-H, Zhang J-HJ-H, Chuang W-CW-C, Yu K-HK$\mathrm{H}$, Huang X-BX-B, Lee Y-CY-C et al. An in vitro study on the effect of combined treatment with photodynamic and chemical therapies on Candida albicans. Int $\mathrm{J}$ Mol Sci 2018, 19(2).

35. Da Silva FC, Fernandes Rodrigues PLPL, Santos Dantas Araújo T, Sousa Santos M, de Oliveira JM, Pereira Rosa L et al. Fluorescence spectroscopy of Candida albicans biofilms in bone cavities treated with photodynamic therapy using blue LED $(450 \mathrm{~nm})$ and curcumin. Photodiagnosis Photodyn Ther 2019;26:366-70.

36. Ma J, Shi H, Sun H, Li J, Bai Y. Antifungal effect of photodynamic therapy mediated by curcumin on Candida albicans biofilms in vitro. Photodiagnosis Photodyn Ther 2019;27:280-7.

37. Rocha MP, Ruela ALM, Rosa LP, Santos GPO, Rosa FCS. Antimicrobial photodynamic therapy in dentistry 
using an oil-in-water microemulsion with curcumin as a mouthwash. Photodiagnosis Photodyn Ther 2020;32:101962.

38. Carmello JC, Pavarina AC, Oliveira R, Johansson B. Genotoxic effect of photodynamic therapy mediated by curcumin on Candida albicans. FEMS Yeast Res 2015, 15(4).

39. Sakima VT, Barbugli PA, Cerri PS, Chorilli M, Carmello JC, Pavarina AC et al. Antimicrobial Photodynamic Therapy Mediated by Curcumin-Loaded Polymeric Nanoparticles in a Murine Model of Oral Candidiasis. Molecules 2018, 23(8).

40. Lee W, Lee DG. An antifungal mechanism of curcumin lies in membrane-targeted action within Candida albicans. IUBMB Life 2014.

41. Garcia-Rubio R, de Oliveira HC, Rivera J, TrevijanoContador N. The Fungal Cell Wall: Candida, Cryptococcus, and Aspergillus Species. Frontiers in Microbiology 2020.

42. Lindberg E, Hammarström H, Ataollahy N, Kondori N. Species distribution and antifungal drug susceptibilities of yeasts isolated from the blood samples of patients with candidemia. Sci Rep 2019.

43. Andrade JT, Fantini de Figueiredo G, Cruz LF, Eliza de Morais S, Souza CDF, Pinto FCH et al. Efficacy of curcumin in the treatment of experimental vulvovaginal candidiasis. Rev Iberoam Micol 2019.

44. Tsao SM, Yin MC. Enhanced inhibitory effect from interaction of curcumin with amphotericin B or fluconazole against Candida species. J Food Drug Anal 2000.

45. Ricatto LGO, Conrado LAL, Turssi CP, França FMG, Basting RT, Amaral FLB. Comparative evaluation of photodynamic therapy using LASER or light emitting diode on cariogenic bacteria: An in vitro study. Eur J Dent 2014.

46. Rios A, He J, Glickman GN, Spears R, Schneiderman ED, Honeyman AL. Evaluation of photodynamic therapy using a light-emitting diode lamp against enterococcus faecalis in extracted human teeth. J Endod 2011.

47. Baltazar LM, Ray A, Santos DA, Cisalpino PS, Friedman AJ, Nosanchuk JD. Antimicrobial photodynamic therapy: An effective alternative approach to control fungal infections. Frontiers in Microbiology 2015.

48. Najafi S, Sheykhbahaei N, Khayamzadeh M, Gholizadeh $\mathrm{N}$. The effect of low level laser on number of Candida albicans colonies In-vitro: A new finding. BMC Oral Health 2019.

49. Wiench R, Skaba D, Stefanik N, Kępa M, Gilowski Ł, Cieślar $\mathrm{G}$ et al. Assessment of sensitivity of selected Candida strains on antimicrobial photodynamic therapy using diode laser $635 \mathrm{~nm}$ and toluidine blue - In vitro research. Photodiagnosis Photodyn Ther 2019.

50. Tan Y, Leonhard M, Moser D, Ma S, Schneider-Stickler B. Antibiofilm efficacy of curcumin in combination with 2-aminobenzimidazole against single- and mixed-species biofilms of Candida albicans and Staphylococcus aureus. Colloids Surfaces B Biointerfaces 2019.

51. Chen E, Benso B, Seleem D, Ferreira LEN, Pasetto S, Pardi $\mathrm{V}$ et al. Fungal-Host Interaction: Curcumin Modulates Proteolytic Enzyme Activity of Candida albicans and Inflammatory Host Response in vitro. Int J Dent 2018.

52. Nosratzehi T, Nosratzehi M, Nosratzehi S, Lotfi F. The comparison of the effect of curcumin with nystatin on inhibition level of Candida albicans. J Exp Pharmacol 2019.

53. Garcia-Gomes AS, Curvelo JAR, Soares RMA, FerreiraPereira A. Curcumin acts synergistically with fluconazole to sensitize a clinical isolate of Candida albicans showing a MDR phenotype. Med Mycol 2012.

54. Neelofar K, Shreaz S, Rimple B, Muralidhar S, Nikhat M, Khan LA. Curcumin as a promising antiCandidal of clinical interest. Can J Microbiol 2011.

55. Shep D, Khanwelkar C, Gade P, Karad S. Safety and efficacy of curcumin versus diclofenac in knee osteoarthritis: A randomized open-label parallel-arm study. Trials 2019.

56. Mansouri K, Rasoulpoor S, Daneshkhah A, Abolfathi S, Salari N, Mohammadi M et al. Clinical effects of curcumin in enhancing cancer therapy: A systematic review. BMC Cancer 2020. 